\title{
Indirect assortative mating for human disease and longevity
}

\author{
Konrad Rawlik $\mathbb{B}^{1} \cdot$ Oriol Canela-Xandri $\mathbb{1}^{1,2} \cdot$ Albert Tenesa $\mathbb{( i D}^{1,2}$
}

Received: 12 June 2018 / Revised: 3 January 2019 / Accepted: 5 January 2019 / Published online: 5 February 2019

(C) The Genetics Society 2019

\begin{abstract}
Phenotypic correlations among partners for traits such as longevity or late-onset disease have been found to be comparable to phenotypic correlations in first-degree relatives. How these correlations arise in late life is poorly understood. Here we introduce a novel paradigm to establish the presence of indirect assortment on factors correlated across generations, by examining correlations between parents of couples, i.e., in-laws. Using correlations in additive genetic values we further corroborate the presence of indirect assortment on heritable factors. Specifically, using couples from the UK Biobank cohort, we show that longevity and disease history of the parents of White British couples are correlated, with correlations of up to 0.09. The correlations in parental longevity are replicated in the FamiLinx cohort, a larger and geographically more diverse historical ancestry dataset spanning a broader time frame. These correlations in parental longevity significantly (pval< 0.0093 for all pairs of parents) exceed what would be expected due to variations in lifespan based on year and location of birth. For cardiovascular diseases, in particular hypertension, we find significant correlations $(r=0.028$, pval $=0.005)$ in genetic values among partners, supporting a model where partners assort for risk factors to some extent genetically correlated with cardiovascular disease. Partitioning the relative importance of indirect assortative mating and shared common environment will require large, well-characterized longitudinal cohorts aimed at understanding phenotypic correlations among none-blood relatives. Identifying the factors that mediate indirect assortment on longevity and human disease risk will help to unravel factors affecting human disease and ultimately longevity.
\end{abstract}

\section{Introduction}

Partner correlations for a variety of phenotypes have been reported when examining environmental and genetic contributions to complex traits (Anonymous 1903; HippisleyCox et al. 2002; Silventoinen et al. 2003; Zietsch et al. 2011; Tenesa et al. 2015; Conley et al. 2016; Hugh-Jones et al. 2016; Muñoz et al. 2016; Nordsletten et al. 2016; Stulp et al. 2016; Xia et al. 2016). These correlations between nominally unrelated individuals are substantial,

Supplementary information The online version of this article (https:// doi.org/10.1038/s41437-019-0185-3) contains supplementary material, which is available to authorized users.

\section{Albert Tenesa}

Albert.Tenesa@ed.ac.uk

1 The Roslin Institute, Royal (Dick) School of Veterinary Studies, The University of Edinburgh, Easter Bush Campus, Midlothian, Edinburgh EH25 9RG, Scotland

2 MRC HGU at the MRC IGMM, Western General Hospital, University of Edinburgh, Crewe Road South, Edinburgh EH4 2XU, UK with magnitude comparable to correlations between firstdegree blood relatives, for instance, between parents and children (Muñoz et al. 2016; Xia et al. 2016). Such effects can be interpreted as phenotypic convergence among partners due to the environmental factors that partners share during their co-habitation. In the case of late-onset diseases and longevity, which are not directly observable or present at the time of mate choice, this would arguably be the simpler explanation. Alternatively, partner correlations for late-onset disease and longevity could arise due to indirect assortative mating. That is, direct assortative mating for traits, characteristics or social factors that are risk factors of disease and potentially observable at the time partners met (for instance, behavioural risk factors of disease such as smoking) would lead to indirect assortative mating for other focal traits, such as longevity or late-onset disease. Here we take direct assortative mating to refer, in general, to nonrandom mate choice based on expressed phenotypes. In particular, we do not distinguish between mate choice that leads to positive or negative phenotypic correlations, the latter often being referred to as dissortative mating. The distinction between the causes that underpin partner effects has implications for the study of human behaviour, 


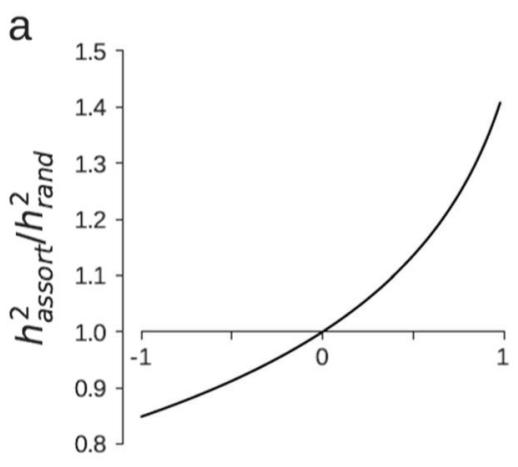

$\rho_{\text {couple }}$ b

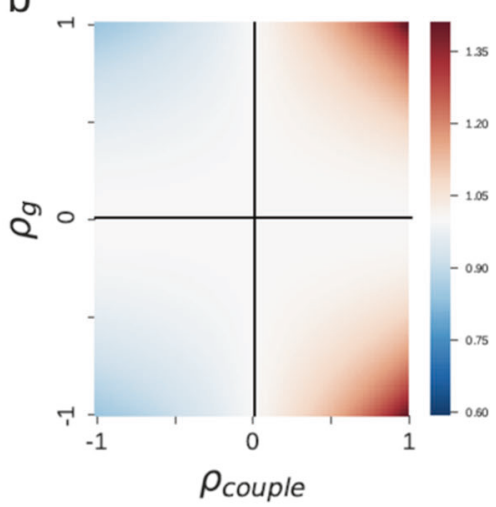

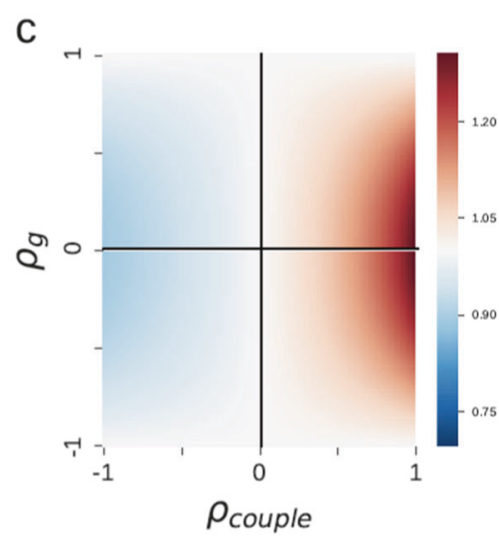

Fig. 1 Effects of indirect assortative mating on heritability and correlations based on the model of (Gianola 1982) (see Supplementary Methods). We consider a pair of traits. One trait that is the target of assortment, e.g., BMI, and a genetically correlated focal trait, e.g., hypertension disease liability. Both traits are taken to have heritabilities of 0.3 in a random mating population. We illustrate relative changes in three genetic parameters as functions of the strength of assortative mating $\left(\rho_{\text {couple }}\right)$ and genetic correlation in a random mating population between the traits $\left(\rho_{\mathrm{g}}\right)$. Specifically, a changes in heritability of the assortment trait, $\mathbf{b}$ changes in heritability of the focal trait

epidemiology and population genetics. It provides information about human mate choice behaviour and informs about the importance of environmental risk factors shared by couples in the household. The importance to population genetics arises, because assortative mating for heritable traits induces a correlation of genetic values among partners, whereas assortment on environmental factors (e.g., social homogamy) and environmental effects shared by partners do not. The correlation of the genetic values of the partners in turn affects the amount of genetic variance of the trait assorted on. As a consequence, estimates of heritability reported in the literature that do not account for assortment overestimate the heritability for that trait in a random mating population due to the covariance among alleles at different loci (Falconer and Mackay 1996) (Fig. 1a, Supplementary Methods). Furthermore, assortative mating for a trait would also induce an increase in heritability for genetically correlated traits (Gianola 1982) (Fig. 1b) and a change in the genetic correlation between the assortment and focal traits (Fig. 1c). This is the case even if these focal traits do not directly underlie mate choice or do not manifest at the time of mate choice. For instance, assortment for body mass index (BMI) would induce an indirect increase in the genetic variance of cardiovascular disease, because there is a positive genetic correlation between these two traits (Bulik-Sullivan et al. 2015) and an increase in their genetic correlation with respect to what would be expected under random mating.

Establishing assortative mating directly requires knowledge of the phenotype at the time of mate choice. Even for phenotypes that are observable at mate choice, such as and $\mathbf{c}$ changes in genetic correlation between the traits. In all three panels, we plot the ratios of the parameter under assortment to random mating. We assume a population at equilibrium after assortative mating (which happens only after a few generations of assortment) relative to a random mating population. In $\mathbf{b}$ and $\mathbf{c}$, colours indicate the ratios of $h^{2}$ or $\rho_{\mathrm{g}}$ in the two populations. Specifically, red colours indicate areas where assortative mating leads to increased heritability in the focal trait and increased absolute genetic correlations, i.e., the ratio of $h^{2}$ or $\rho_{\mathrm{g}}$ after assortative mating to that in a random mating population is greater than one

height, such data are rare. For phenotypes such as longevity or disease risk, which only manifest long after mate choice, such data can obviously not be collected. Recent work, starting with Tenesa et al. (2015), has therefore concentrated on using genotype information to establish assortment (Robinson et al. 2017). As genetic values (i.e., polygenic scores) are fixed at birth, correlations between partners in such values provides direct evidence for assortment. However, this approach is limited by how well genetic values predict phenotype, i.e., the heritability, and the precision with which genetic values can be estimated. The heritabilities of longevity and many late-onset diseases are medium to low (Canela-Xandri et al. 2018), with estimates for single-nucleotide polymorphism (SNP) heritability of longevity ranging from 0.12 to 0.3 (Kaplanis et al. 2018). Furthermore, numbers of disease cases, for many diseases that are rare in the general population, and individuals with lifespan information are small in large, prospectively collected and genotyped cohorts such as UK Biobank, limiting the precision of estimates of genetic values.

Here we propose a related alternative approach. We examine correlations between the parents of partners. That is, between the father of one spouse and the father of the partner. We present data showing that there is indirect assortment for both longevity and risk of disease. Specifically, we find that humans choose partners with similar parental history of disease and parental longevity. As partner choice most likely happens before the parental onset of most of these diseases or parental death, these are unlikely to be the traits on which such choice is made. Furthermore, 


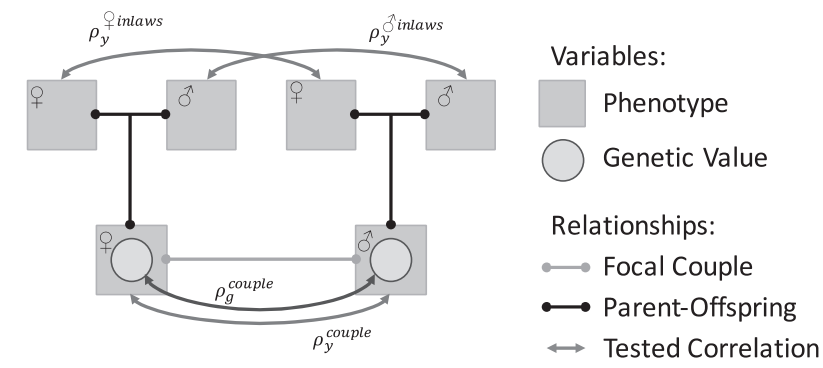

Fig. 2 Schematic outline of the study. We consider couples and their parents. We compute phenotypic correlations between couples $\left(\rho_{\mathrm{y}}{ }^{\text {couple }}\right)$ for longevity and disease status. Such correlations could be explained by the couple sharing a nuclear environment, e.g., shared exposures in the shared home or shared diet. To exclude the possibility of convergence based on shared nuclear environment, we examined parental correlations, i.e., correlations between the fathers $\left(\rho_{\mathrm{y}}{ }^{\text {dinlaws }}\right)$ and mothers $\left(\rho_{\mathrm{y}}^{\text {Pinlaws }}\right)$ of the partners. Such correlations cannot arise due to the nuclear couple environment, but require non-random mating and across-generation correlations. The across-generation correlations could arise due to heritable genetic effects or culturally transmitted environmental effects. We therefore also examined correlations in genetic values $\left(\rho_{\mathrm{g}}^{\text {couple }}\right)$, which provide evidence for non-random mating with respect to heritable factors

as these traits are correlated across generations, indirect assortment is the most parsimonious model. Finally, we demonstrate assortment directly, showing that the genetic values (i.e., genomic best linear unbiased predictors (GBLUPs)) for hypertension are correlated among partners. Given that assortment for hypertension itself is unlikely, we hypothesize that this correlation in genetic values arises through assortment for one or more traits that influence mate choice and which are genetically correlated with hypertension.

\section{Materials and methods}

The general framework of this study is outlined in Fig. 2 . We investigated partner correlations $\left(\rho_{y}{ }^{\text {couple }}\right)$ in longevity (see Partner Correlations for Longevity). To dissect the source of these correlations and, in particular, to establish whether they arise due to indirect assortment, we followed several approaches. First, we considered correlations in longevity between parents of focal partners $\left(\rho_{\mathrm{y}}{ }^{\text {Pinlaws }}\right.$ and $\rho_{\mathrm{y}}{ }^{\text {Jinlaws }}$ ) (see Parental Correlations of Longevity). That is, $\rho_{\mathrm{y}}{ }^{\text {dinlaws }}$ is the correlation between the two fathers of a husband and wife pair. Then, we considered to what extend potential targets of assortment, such as BMI or socioeconomic status, which are correlated across generations, explained any observed parental correlations (see Effect of environmental factors on parental correlations in longevity). Finally, we evaluated correlations between genetic values (GBLUPs) of the focal partners $\left(\rho_{\mathrm{g}}{ }^{\text {couple }}\right)$ to demonstrate assortment directly (see Partner correlations of genetic values of parental longevity).

We hypothesized that indirect assortative mating for longevity could be driven by assortative mating for disease risk factors. We therefore also examined indirect assortment on disease risk, following the same approaches as for longevity (see Parental correlations in disease history).

The majority of analyses were performed using data from the UK Biobank cohort, but where possible results were replicated using the FamiLinx cohort (Kaplanis et al. 2018).

\section{Couples in the UK Biobank cohort}

Identification of heterosexual couples in the UK Biobank has been previously reported (Tenesa et al. 2015). Specifically, using household sharing information we identified a set of 105,380 households with exactly two members in the cohort. Of these, 90,297 satisfied all of the following criteria: (a) individuals reported different ages for one or both parents; (b) individuals had an age difference of $<10$ years; (c) individuals were of opposite gender; (d) both individuals reported to live only with their partner or partner and children. We restricted our analysis to a subset of 79,094 couples for which both partners self-reported to be of White-British ethnicity.

\section{Couples in the Familinx cohort}

The FamiLinx cohort (Kaplanis et al. 2018), consisting of $86,124,644$ individuals, is based on publicly accessible genealogy data ranging back up to the early fifteenth century and covering individuals born across the world, although individuals of European and North American birth dominate. In our analysis we restricted ourselves to a subset of individuals with full information regarding year of birth and death, latitude and longitude of the birth location. We removed individuals with a birth location along the zero meridian, as visual inspection suggested a majority of these to be coding errors. We furthermore removed individuals with lifespans below 30 years or above 130 years. Furthermore, following previous analysis (Kaplanis et al. 2018), we removed those individuals born before 1600 due to the sparsity and lower reliability of data before that date, and those individuals born after 1910 due to the bias towards individuals with reduced lifespan after that date. Finally, also following previous analysis (Kaplanis et al. 2018), we removed individuals who died during the American Civil War (year of death 1861-1865), the first World War (year of death 1914-1918) and the second World War (year of death 1939-1945) due to the excess number of early death in these periods. This resulted in a dataset of 3,445,971 individuals. Considering individuals 
with common offspring, we identified a set of 239,541 couples.

\section{Definition of birth location}

Both the UK Biobank and FamiLinx contain information about the birth locations of individuals, which we used to adjust for any potential geographical differences in longevity. However, in both cohorts the provided information is at a scale too fine to allow for effective stratification based on birth location. We therefore defined a birth location at a coarser scale in both cohorts.

The UK Biobank contains information about the coordinates of the birth location with a resolution of $1 \mathrm{~km}$. We identified a subset of individuals with miscoded coordinates corresponding to birth in the Atlantic Ocean identified through visual inspection and set their birth location as missing. We used a $15 \mathrm{~km}$ grid to define birth location. That is, we assigned all individuals who share birth coordinates when divided by $15 \mathrm{~km}$ and rounded to an integer to the same birth location.

In the FamiLinx cohort, we defined a $1^{\circ}$ latitude and longitude grid to derive birth location.

\section{Genotypes and estimation of genetic values in UK Biobank}

To perform genetic analyses we identified a set of qualitycontrolled, genotypically White-British individuals from the UK Biobank. Using appropriate subsets of these individuals as described for specific analyses, we jointly estimated SNP heritabilities and SNP effects following the mixed model approach using the DISSECT tool (Canela-Xandri et al. 2015). We used the estimated SNP effects to compute genetic values (i.e., GBLUPs). All models included the leading 20 genomic principal components as fixed effects.

The set of individuals available for genetic analyses was identified as follows. We used the data for the individuals genotyped in phase 1 of the UK Biobank genotyping programme. A total of 49,979 individuals were genotyped using the Affymetrix UK BiLEVE Axiom array and 102,750 individuals using the Affymetrix UK Biobank Axiom array. Details regarding genotyping procedure and genotype-calling protocols are provided elsewhere (http:// biobank.ctsu.ox.ac.uk/crystal/refer.cgi?id =155580). We performed quality control using the entire set of genotyped individuals before extracting the White-British cohort used in our analyses. From the overlapping genetic markers between the two arrays, we excluded those which were multi-allelic, their overall missingness rate exceeded $2 \%$ or which exhibited a strong platform specific missingness bias (Fisher's exact test, pval $<10^{-100}$ ). We also excluded individuals if they exhibited excess heterozygosity, as identified by UK Biobank internal quality control (QC) procedures (http://biobank.ctsu.ox.ac.uk/crystal/refer.cgi?id $=155580$ ), if their missingness rate exceeded $5 \%$ or if their self-reported sex did not match genetic sex estimated from $\mathrm{X}$ chromosome inbreeding coefficients. These criteria resulted in a reduced dataset of 151,532 individuals. To define the genotypically White-British subset, we performed a Principal Components Analysis of all individuals passing genotypic QC using a linkage disequilibrium pruned set of 99,101 autosomal markers (http://biobank. ctsu.ox.ac.uk/crystal/refer.cgi?id $=149744$ ), which passed our SNP QC protocol. The genotypically White-British individuals were defined as those for whom the projections onto the leading 20 genomic principal components fell within three SDs of the mean and who self-reported their ethnicity as White-British. We furthermore pruned the set of genotypically White-British individuals removing one individual from pairs with relatedness above 0.0625 (corresponding to second degree cousins), to obtain a dataset of unrelated genotypically White-British individuals. Finally, in our genetic models we only used genetic variants that had passed QC, that did not exhibit departure from Hardy-Weinberg equilibrium (pval $<10^{-50}$ ) in the unrelated genotypically White-British cohort and which had a minor allele frequency $>5 \%$.

\section{Partner correlations for longevity}

We estimated partner correlations of longevity, defined as the age in years at death using data from the two cohorts: the UK Biobank and Familinx. We also computed correlations of longevity adjusted for cohort effects. Specifically, we computed adjusted longevity as the difference between an individual's lifespan and the mean lifespan of the stratum defined by the individual's sex, birth year and birth location (see Definition of birth location), excluding all strata with fewer than 10 individuals.

As the majority of UK Biobank participants are alive, we used the biological mothers and fathers of participants. Specifically, we identified self-reported White-British individuals with both parents deceased (using data fields UKBID 21000, 1797 and 1835) and non-missing birth location (see Definition of birth location). This yielded 252,899 pairs of parents for which we computed Pearson's correlations between longevity extracted from data fields UKBID 1807 and 3526. The UK Biobank does not directly contain information regarding the years or location of birth of parents of participants. As such, we used the participant's place and year of birth (UKBID 34) as proxy measures of the parent's place and year of birth. For a subset of parents, specifically parents who are still alive at recruitment of the 
participant, we can infer the parents' year of birth from the date of recruitment and the parents' age. The subset of parents who are still alive is relatively small, only $22 \%$ of fathers and 39\% mothers, respectively, and is complementary to the set of parents used in the analysis, who were required to be deceased. Although we can therefore not use the data in our analysis, it allows us to evaluate the effect of using a proxy measure. The correlation between the year of birth of the offspring and their parent is relatively high with $\rho=0.78$.

In the FamiLinx cohort, we used all 239,541 couples identified as described above (see Couples in the FamiLinx cohort). We computed longevity as the difference of year of death and year of birth.

\section{Parental correlations of longevity}

We computed Pearson's correlations of longevity and adjusted longevity for parents of partners. That is, we computed, e.g., the correlation between the longevity of the two fathers of the male and female partners in a couple. We considered the three combinations of parents, i.e., the two fathers or the two mothers of the partners and the father of one partner and the mother of the other partner, separately. Both longevity and adjusted longevity were computed as for the analysis of partner correlations (see Partner correlations for longevity).

Of the 79,094 couples identified in the UK Biobank (see Couples in the UK Biobank) 40,504 had both mothers and 60,978 both fathers deceased, whereas there were 104,922 father-mother pairs. Among the 3,445,971 individuals retained for analysis in the FamiLinx cohort (see Couples in the FamiLinx cohort), we identified 97,223 sets of fathers, 66,077 sets of mothers and 143,896 father-mother pairs.

We computed expected distributions of parental correlations due to geographical and temporal mating structure in the population based on permutations. Specifically, we generated fictitious sets of couples, which matched the observed mating structure for birth years and birth locations, and computed the parental correlations in longevity for these fictitious couples. To generate the fictitious couples we stratified couples based on the birth year and birth locations of both partners and permuted male partners within each stratum. To allow for effective permutations we only included couples in strata of size larger than 10 in the analysis. For each permutation, we computed Pearson's correlations of parental longevity as a test statistic. Empirical $p$-values were then computed as the fraction of statistics exceeding the statistic computed without permutation, based on 10,000 permutations.

\section{Effect of environmental factors on parental correlations in longevity}

We evaluated partner correlations for a range of potential assortment factors and evaluated their contribution to any observed correlations in parental longevity.

Specifically, we extracted Townsend Deprivation Index (UKBID 189), height (UKBID 50), waist-to-hip ratio (computed from UKBID 48 and 49), BMI (UKBID 21001) and smoking history in pack years (UKBID 20161) for all individuals in the 79,094 couples identified in the UK Biobank. The Townsend Deprivation Index is an area measure of socio-economical deprivation. We computed Pearson's correlations between the male and female partners for all pairs of these variables as well as birth year.

We then computed linear regression models, regressing parental longevity on birth year, birth location, as well as Townsend Deprivation Index and height, waist-to-hip ratio, BMI and smoking history in pack years, and the squares of these factors, of their children. Birth year and birth location were coded as categorical variables, whereas all other factors and their squares were included as continuous variables. Using the fitted models, we computed residuals and correlations between couples using these residuals. Comparing these, we quantified the change in correlations due to inclusion of individual covariates in the models.

\section{Partner correlations of genetic values of parental longevity}

As the majority of individuals in the UK Biobank are still alive, we cannot estimate genetic values for longevity directly. We therefore again use information about the lifespans of parents of participants and estimate genetic values (GBLUPs) for parental longevity as a proxy for genetic values of an individual's longevity.

Of the UK Biobank individuals retained for genetic analysis (see Genotypes and estimation of genetic values in UK Biobank), subsets of 79,216 and 64,002 had respectively deceased fathers and mothers. Using these individuals, we estimated SNP heritabilities and genetic variant effects for parental longevity based on common variants, i.e., variants with minor allele frequency above $5 \%$. Of the 79,094 couples identified in the UK Biobank (see Couples in the UK Biobank cohort) a subset of 10,160 couples consisted of individuals retained for genetic analysis. For these couples, using the estimated genetic variant effects, we computed genetic values (Canela-Xandri et al. 2015, 2016) for parental longevity and computed their Pearson's correlation. 


\section{Disease history in the UK biobank}

Participants in the UK Biobank provide information about the family history for 12 diseases for both biological parents (UKBID 20107 and 20110). Considering the 79,094 couples identified in the UK Biobank (see Couples in the UK Biobank cohort), disease history for both biological parents of each partner was reported by 58,043 couples for heart disease, stroke, chronic bronchitis, high blood pressure, diabetes and Alzheimer's disease, and by 57,644 couples in the case of lung cancer, bowel cancer, Parkinson's disease and depression. For the latter subset, information regarding disease history for the relevant parent for breast and prostate cancer was available for each partner.

The twelve disease for which family history was provided do not directly match disease reported in the selfreported medical history of participants (UKBID 20002). To identify self-reported controls, the methodology of Muñoz et al. (2016) was utilized, to match diseases to those reported for family history.

\section{Parental correlations in disease history}

Following the methods for parental correlations for longevity (see Parental correlations of longevity), we computed correlations of disease history between the fathers and mothers of couples in the UK Biobank. We also computed correlations for each disease using only couples where both partners are self-reported controls for the relevant disease.

As disease history or status for an individual is a binary trait, Pearson's correlations are not a suitable measure of correlation. Instead we computed polychoric correlations (Drasgow 1986) using the R package polycor (Fox 2010). In addition, we assessed dependence between partner's family histories using a $x^{2}$ test and by computing empirical mutual information (Cover and Thomas 2012). For mutual information we computed an empirical $p$-value for departure from independence using permutations. That is, we computed empirical mutual information for 1000 datasets in which family history for the male partners had been permuted and compared them with the empirical mutual information on the observed data.

As for longevity, we evaluated the expected effect of assortment due to place and year of birth using permutations. Permutations were performed as for longevity, using the $x^{2}$ statistics, rather than Pearson's correlation, as test statistic.

We performed an additional permutation analysis to assess the impact of using the offspring's year of birth as a proxy for the parents' year of birth. Unlike in the analysis of longevity, where all parents are deceased, a subset of parents with family history is still alive. For these parents we can compute the year of birth. On the subset of parents with available year of birth, we permuted UK Biobank couples within the years of birth of their parents. That is, the offspring within the years of birth of the parents. We did not permute within both birth year and birth location strata due to the smaller sample size.

\section{Partner correlations of genetic values of disease history}

We computed correlations for genetic values of parental disease history and self-reported disease status. For own disease status, we restricted the analysis to diseases with prevalence in the sample above $5 \%$ and excluding prostate and breast cancers.

For family disease history traits, we fitted models with only genomic principal components and models that also included the participant's birth year and birth location as categorical and the parents' age as continuous covariates. The parent's age was computed as either the age at death (UKBID 1807 and 3526), if the parent was deceased, or age at assessment (UKBID 2946 and 1845) otherwise. Models used to estimate genetic values for self-reported disease also included the participant's sex, age and Townsend Deprivation Index as fixed effects.

We fitted models using all individuals available for genetic analysis (see Genotypes and estimation of genetic values in UK Biobank), who reported family history. We transformed heritabilities that were estimated on the observed scale, i.e., modelling disease status directly, to the liability scale using the sample-specific prevalence (Lee et al. 2011). Using SNP effects estimated on all individuals, we computed genetic values for the 10,160 couples that comprised individuals retained for genetic analysis (see Genotypes and estimation of genetic values in UK Biobank) and computed their Pearson's correlations. We combined paternal and maternal estimates using the Olkin-Pratt fixed effect approach (Schulze 2004).

\section{Results}

\section{Partner correlations in longevity}

We found that the lifespan of the biological mothers and fathers of all self-reported White-British individuals in the UK Biobank with both parents deceased was correlated and significantly different from $0\left(\rho_{\mathrm{y}}{ }^{\text {couple }}=0.11,95 \%\right.$ confidence interval (CI) $0.107-0.114$, pval $<10^{-188}$ ). The correlation was only slightly reduced $\left(\rho_{\text {y-adj }}{ }^{\text {couple }}=0.10,95 \%\right.$ CI $0.091-0.108$, pval $<10^{-188}$ ) and remained significantly different from 0 when adjusting for the participants' year of birth as a proxy of the parent's year of birth, which itself was unavailable. This finding reproduced in the FamiLinx 
cohort. Specifically, although partner correlations for longevity in the FamiLinx cohort were significantly higher $\left(\rho_{\mathrm{y}}{ }^{\text {couple }}=0.18,95 \%\right.$ CI $0.176-0.183$, pval $\left.<10^{-188}\right)$, correlations for lifespans adjusted for an individual's year and place of birth were comparable to those in the UK Biobank cohort $\left(\rho_{\text {y-adj }}{ }^{\text {couple }}=0.125,95 \%\right.$ CI $0.121-0.129$, pval $\left.<10^{-188}\right)$.

\section{Parental correlations of longevity}

We found significant correlations for the lifespans of both mothers $\left(\rho_{\mathrm{y}}{ }^{\text {Pinlaws }}=0.049,95 \%\right.$ CI $0.038-0.062$, pval $\left.=10^{-15}\right)$ and fathers $\left(\rho_{\mathrm{y}}{ }^{\text {dinlaws }}=0.032, \quad 95 \% \quad \mathrm{CI}\right.$ $0.022-0.042$, pval $=10^{-10}$ ) of couples in the UK Biobank. This finding reproduced in the FamiLinx cohort. Although we again observed higher correlations in lifespans of mothers $\left(\rho_{\mathrm{y}}^{\text {Pinlaws }}=0.061,95 \%\right.$ CI $0.053-0.068$, pval $\left.=10^{-55}\right)$ and fathers $\left(\rho_{\mathrm{y}}{ }^{\text {dinlaws }}=0.071,95 \% \mathrm{CI}\right.$ $0.064-0.077$, pval $=10^{-107}$ ) of couples compared with the UK Biobank, correlations between adjusted lifespans were again comparable to those in the UK Biobank $\left(\rho_{\mathrm{y} \text {-adj }}\right.$ finlaws $=0.02,95 \%$ CI $0.012-0.030$, pval $=10^{-7}$ and $\rho_{\text {y-adj }}{ }^{\text {dinlaws }}$ $=0.03,95 \%$ CI $0.023-0.038, \mathrm{pval}=10^{-17}$ for mothers and fathers, respectively). Considering father-mother pairs, we observed reduced correlations in the UK Biobank

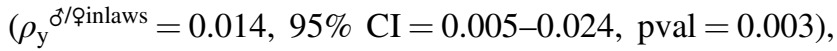
which however were still significant. In the Familinx cohort, on the other hand, correlations for father-mother pairs were comparable to those between fathers and mothers, and significant $\left(\rho_{\mathrm{y}}{ }^{\mathrm{d} /}{ }^{\text {inlaws }}=0.055,95 \%\right.$ CI $0.049-0.060$, $\mathrm{pval}=10^{-15} \quad$ and $\quad \rho_{\mathrm{y} \text {-adj }}{ }^{\mathrm{d} / \text { qinlaws }}=0.055, \quad 95 \% \quad \mathrm{CI}$ $0.049-0.060$, pval $=10^{-15}$ for observed and adjusted lifespan, respectively). We did not consider father-mother correlations in the UK Biobank cohort further and discuss the likely reasons for the observed discrepancy below (see Discussion).

We compared the observed parental correlations with the distribution of correlations for fictitious sets of couples with matched mating structure for year and location of birth. The expected correlation due to mating structure, i.e., the mean correlation across fictitious sets of couples, were small and not significantly different from 0 in the UK Biobank $\left(\rho_{\text {mean }}\right.$ $=0.02, \mathrm{SD} 0.006$ and $\rho_{\text {mean }}=0.01, \mathrm{SD} 0.005$ for mothers and fathers, respectively). Expected correlations were larger and significantly different from 0 in the FamiLinx cohort $\left(\rho_{\text {mean }}=0.03, \mathrm{SE} 0.007, \rho_{\text {mean }}=0.03, \mathrm{SD} 0.005\right.$ and $\rho_{\text {mean }}$ $=0.02$, SD 0.004 for mother, father, and mother-father pairs, respectively). The observed correlations lie in the extreme tails of the distributions of correlations between parents' lifespans (Supplementary Figure S1). The empirical $p$-values for the observed correlations are 0.0002 and < 0.0001 for mothers of couples in UK Biobank and FamiLinx, respectively, and 0.0093 and $<0.0001$ for the fathers of couples in UK Biobank and FamiLinx, respectively. For father-mother pairs of couples in the FamiLinx cohort, the empirical $p$-value for the observed correlation is $<0.0001$.

Year and birth place, socio-economic status (as measured by Townsend Deprivation Index), height, waist-to-hip ratio, BMI and smoking history measured in pack years (as proxies of a putative behavioural factor associated with disease and longevity), showed significant partner correlations in the UK Biobank (Supplementary Table S1). Adjusting parental lifespans for any of these factors reduced the observed correlations. Birth year and location were the most important factors, reducing the observed correlations for both maternal and paternal longevity by around 55\%. Socio-economic status and the other factors had a lesser but still important effect on the correlation of lifespan of parents, reducing such correlation an additional $\sim 15 \%$.

Significant SNP heritabilities were observed for mother's $\left(h^{2}=0.03,95 \%\right.$ CI $\left.0.02-0.04\right)$ and father's $\left(h^{2}=0.04,95 \%\right.$ CI 0.03-0.05) longevity (Supplemental Table S3). These SNP heritabilities for a parental phenotype are under certain assumptions expected to be half the SNP heritability of the phenotype measured in the individual. Correlations between partners in genetic values of parental longevity were not found to be significantly different from $0\left(\rho_{\mathrm{g}}\right.$ couple $=-0.007$, $95 \% \mathrm{CI}-0.026$ to 0.013 , pval $=0.5$ and $\rho_{\mathrm{g}}{ }^{\text {couple }}=0.01$, $95 \% \mathrm{CI}-0.009$ to 0.030 , pval $=0.3$ for paternal and maternal longevity, respectively).

Table 1 Polychroic correlations for family history of fathers and mothers of couples in the UK Biobank

\begin{tabular}{|c|c|c|c|c|c|c|}
\hline & \multicolumn{3}{|c|}{ Father $\left(\rho_{\mathrm{y}}{ }^{\text {dinlaws }}\right)$} & \multicolumn{3}{|c|}{ Mother $\left(\rho_{\mathrm{y}}^{\text {Pinlaws }}\right)$} \\
\hline & $\rho_{\text {chor }}$ & SE & $P$ & $\rho_{\text {chor }}$ & SE & $P$ \\
\hline Heart disease & 0.04 & 0.006 & $6 \times 10^{-11}$ & 0.07 & 0.007 & $9 \times 10^{-23}$ \\
\hline Stroke & 0.02 & 0.009 & 0.003 & 0.06 & 0.009 & $2 \times 10^{-11}$ \\
\hline Lung cancer & 0.04 & 0.012 & $1 \times 10^{-4}$ & 0.08 & 0.018 & $1 \times 10^{-5}$ \\
\hline Bowel cancer & 0.04 & 0.015 & 0.009 & -0.01 & 0.017 & 0.747 \\
\hline Breast cancer & - & - & - & 0.01 & 0.012 & 0.325 \\
\hline $\begin{array}{l}\text { Chronic } \\
\text { bronchitis }\end{array}$ & 0.06 & 0.01 & $2 \times 10^{-9}$ & 0.06 & 0.015 & $7 \times 10^{-5}$ \\
\hline $\begin{array}{l}\text { High blood } \\
\text { pressure }\end{array}$ & 0.09 & 0.007 & $1 \times 10^{-35}$ & 0.08 & 0.006 & $7 \times 10^{-38}$ \\
\hline Diabetes & 0.02 & 0.012 & 0.067 & 0.04 & 0.011 & 0.001 \\
\hline Alzheimer's & 0.07 & 0.017 & $2 \times 10^{-5}$ & 0.08 & 0.011 & $3 \times 10^{-13}$ \\
\hline Parkinson's & 0.02 & 0.027 & 0.267 & 0.04 & 0.034 & 0.13 \\
\hline Depression & 0.03 & 0.022 & 0.103 & 0.04 & 0.014 & 0.005 \\
\hline Prostate cancer & 0.04 & 0.013 & 0.004 & - & - & - \\
\hline
\end{tabular}

$\rho_{\text {chor polychoric correlation. } P=\text { p-value for } \rho_{\text {chor }}=0}$ 


\section{Parental correlations of disease history}

We found significant $(P<0.05)$ polychoric correlations, which were consistent for both fathers and mothers, for half of the 12 examined diseases: heart disease, stroke, lung cancer, chronic bronchitis, hypertension, and Alzheimer's disease (Table 1, Supplementary Table S4). Only stroke in fathers failed significance after Bonferroni correction $(P<$ $0.05 / 22$ ). Of these, the largest correlation was for paternal hypertension $\left(\rho_{\mathrm{y}}{ }^{\text {dinlaws }}=0.09,95 \%\right.$ CI $0.08-0.11$, pval $\left.=10^{-35}\right)$ and the smallest for paternal stroke $\left(\rho_{\mathrm{y}}{ }^{\text {dinlaws }}\right.$ $=0.02,95 \%$ CI $0.01-0.04$, pval $=0.003$ ). The history of prostate cancer among fathers of couples was also significantly correlated $\left(\rho_{\mathrm{y}}{ }^{\text {dinlaws }}=0.04,95 \%\right.$ CI $0.01-0.06$, $\mathrm{pval}=0.004)$. Among mothers, the correlations for lung cancer $\left(\rho_{\mathrm{y}}{ }^{\text {Pinlaws: }}=0.08,95 \%\right.$ CI $0.04-0.11$, pval $\left.=10^{-5}\right)$, hypertension $\left(\rho_{\mathrm{y}}{ }^{\text {oinlaws }}=0.08,95 \% \mathrm{CI} 0.07-0.10, \mathrm{pval}<10\right.$ $\left.{ }^{-37}\right)$ and Alzheimer's $\left(\rho_{\mathrm{y}}^{\text {}}{ }^{\text {inlaws }}=0.08,95 \%\right.$ CI $0.06-0.10$, pval $<10^{-12}$ ) were the largest, whereas the correlations for heart disease were only marginally smaller $\left(\rho_{\mathrm{y}}{ }^{\text {Pinlaws }}=0.07\right.$, 95\% CI $0.06-0.09$, pval $<10^{-22}$ ). The analysis using only couples of self-reported controls was largely in agreement with the analysis using all couples (Supplementary Table S5).

We compared the observed parental associations to the distribution of associations for fictitious sets of couples with matched mating structure for year and location of birth (Supplementary Table S6). Results using a mating structure based on the parent's year of birth, available in only a subset of parents, were consistent with the results obtained when using the participant's year of birth as a proxy measure (Supplementary Table S7).

We found modest but significant SNP heritabilities for a majority of the considered parental family histories (Supplementary Table S8). Correlations between genetic values of partners were significant $(P<0.05)$ for maternal and paternal history of hypertension, as well as maternal heart disease, stroke and chronic bronchitis (Table 2). However, only maternal chronic bronchitis and hypertension remained significant after Bonferroni correction $(P<0.05 / 22)$. Although hypertension in fathers did not reach the stringent Bonferroni correction threshold, the size of the correlation was similar to that of maternal hypertension. Furthermore, hypertension remained significant in the meta-analysis of paternal and maternal correlations (Table 2).

Although correlations between genetic values were reduced, when adjusting for an individual's birth year, birth location and the parent's age, they remained significant $(P$ $<0.05$ ) for maternal and paternal hypertension, and maternal chronic bronchitis and stroke (Supplementary Table S9).

Despite the smaller numbers of cases, when using own disease status rather then parental disease history, we again found the correlations of genetic values of partners for
Table 2 Within couple correlations of genetic values $\left(\rho_{\mathrm{g}}{ }^{\text {couple }}\right)$ for family history and self-reported disease in genotyped couples in the UK Biobank

\begin{tabular}{|c|c|c|c|c|c|}
\hline & \multicolumn{2}{|c|}{$\begin{array}{l}\text { Parental family } \\
\text { history }^{\text {a }}\end{array}$} & \multicolumn{3}{|l|}{ Self $^{b}$} \\
\hline & $\rho$ & $P$ & $\rho$ & $95 \% \mathrm{CI}$ & $P$ \\
\hline Hypertension & 0.03 & $8 \times 10^{-6}$ & 0.028 & 0.009 to 0.048 & 0.005 \\
\hline $\begin{array}{l}\text { Chronic } \\
\text { bronchitis }\end{array}$ & 0.019 & 0.07 & 0.011 & $\begin{array}{l}-0.008 \text { to } \\
0.031\end{array}$ & 0.26 \\
\hline Heart disease & 0.016 & $9 \times 10^{-3}$ & -0.015 & $\begin{array}{l}-0.034 \text { to } \\
0.005\end{array}$ & 0.14 \\
\hline Stroke & 0.013 & 0.12 & 0.004 & $\begin{array}{l}-0.016 \text { to } \\
0.023\end{array}$ & 0.7 \\
\hline Diabetes & 0.009 & 0.09 & 0.024 & $0.004-0.043$ & 0.02 \\
\hline Prostate cancer & 0.009 & 0.34 & - & & - \\
\hline Lung cancer & 0.005 & 0.32 & - & & - \\
\hline Alzheimer's & 0.004 & 0.27 & - & & - \\
\hline $\begin{array}{l}\text { Severe } \\
\text { depression }\end{array}$ & 0.003 & 0.41 & 0.017 & $\begin{array}{l}-0.002 \text { to } \\
0.036\end{array}$ & 0.09 \\
\hline Parkinson's & -0.001 & 0.42 & - & & - \\
\hline Breast cancer & -0.004 & 0.68 & - & & - \\
\hline Bowel cancer & -0.008 & 0.14 & - & & - \\
\hline
\end{tabular}

${ }^{\mathrm{a}}$ Meta-analysis of paternal and maternal results, with the exception of prostate cancer and breast cancer, which are paternal and maternal results, respectively, separate results for all disease can be found in Supplementary Table S10,

${ }^{\mathrm{b}}$ Contains only results for self-reported non-sex-specific disease with UK Biobank prevalence $>5 \%, \rho=$ Pearson's correlation between genetic values in couples, $P=p$-value for $\rho=0$

hypertension to be significant and of similar size to the parental hypertension $\left(\rho_{\mathrm{g}}{ }^{\text {couple }}=0.03,95 \%\right.$ CI $0.01-0.05$, pval $=0.005$.

\section{Discussion}

Partner correlations for age at death have been demonstrated going back to early work on assortative mating (Anonymous 1903). We were able to reproduce these results in two independent cohorts of unprecedented sample size. The partner correlations we observed were significantly lower than the correlation of 0.23 reported a century ago for a much smaller sample from the UK (Anonymous 1903), but similar to more recent estimates of 0.12 in a Canadian population (Philippe 1978). The sample of partners from the UK Biobank used here was censored, consisting of parents of participants and necessarily excluding all parents who were still alive. However, the close agreement between estimates in the independent FamiLinx cohort and previous estimates does not suggest that this introduced substantial bias. The results suggest that partner correlations for lifespan, after adjusting for mating structure due to year and 
place of birth, are in the region of 0.1-0.12. Estimates of heritability for longevity in the FamiLinx cohort imply a phenotypic correlation between first-degree relatives of 0.06 (Kaplanis et al. 2018), whereas previous estimates of heritability suggest higher correlations of 0.13 (Herskind et al. 1996). Our estimates of SNP heritability for longevity of an individual's parents suggest a phenotypic correlation between first-degree relatives of 0.03 or 0.04 . Unlike previous estimates, our estimates are based on samples of unrelated individuals, largely precluding inflation due to shared environment that may have affected previous estimates. On the other hand, we only estimate the variance explained by common SNPs and therefore likely underestimate the heritable component of longevity. However, even allowing for the whole range of estimates, partner effects seem to be comparable in magnitude, or even exceed, genetic effects on longevity.

Various possible explanations exist for the observed partner correlations. The year of death of partners could potentially be correlated due to effects directly related to the partner's death (i.e., a partner's death has a causal link with the other partner's death). This together with the assortment by birth year, as we observed in the UK Biobank, would lead to partner correlations for lifespan. More generally, convergence due to shared environmental factors represents in the absence of other data the most plausible explanation for the observed partner correlations. That is, partners share one or more environmental risk factors, e.g., a diet, which affects life expectancy. Such shared environment can be restricted to the partners. More broadly, correlations may reflect mating structure within a broader shared environment. For example, partners may mate preferably in the same socio-economical stratum. This may, depending on interpretation, be considered a form of assortative mating. In particular, one's broader environment may have genetic underpinnings. For example, one's socio-economic status may be influenced by heritable traits such as educational attainment (Belsky et al. 2018) and their combined effect may reduce social mobility.

By comparison with partner correlations, the estimates of correlations between parental longevity we report are substantially smaller. Indeed, they are arguably small enough to be considered practically insignificant. However, we do not argue for their significance based on their magnitude. As a matter of fact, taking into account the low heritability of longevity, they are expected to be small. Instead, their relevance lies in the information their presence provides about the larger partner correlations. They provide evidence that observed partner correlations arise due to a form of assortment. Specifically, they provide evidence that mating is not random with respect to factors, which persist across generations. As the parents of partners do not share the narrow environment of the couple, our results provide evidence that the observed correlations, at least partly, arise due to mating structure related to factors correlated across generations. Correlations across generations can arise due to several distinct pathways, which cannot be differentiated by considering correlations of parents of couples. On the one hand, genetic effects lead to across generation correlations. These can take the form of direct effects, i.e., classical heritability, or indirect parent offspring effects as recently described (Kong et al. 2018). On the other hand, crossgenerational correlations can also arise due to non-genetic transmission, i.e., cultural heritability. For example, low social mobility in a society will lead to parent offspring correlations in socio-economic status.

Similar to partner correlations, parental correlations are expected to be partly explained by differences in life expectancy across history and geography. We have demonstrated that a mating structure based on these factors alone is unlikely to explain the observed correlations. Identification of the specific factors contributing to the observed partner correlations represents an important question for future research. We have examined the contribution of a small number of baseline factors, each of them heritable (Canela-Xandri et al. 2018), including known targets of assortment such as height and factors reflecting social mating structure like the Townsend Deprivation Index. All of the examined factors explain parts of the observed correlation and it does not appear a single factor will be able to explain partner correlations in longevity. However, our results suggest that these factors and socioeconomic status are correlated across generations, as the children's phenotypes and socio-economic status explain some of the correlation in longevity of their respective parents.

We were not able to demonstrate correlations in genetic values for longevity. Lack of such correlations would be consistent with environmental assortment, i.e., mating within a broader shared environment or cultural transmission of factors across generations. However, power to detect correlations in genetic values is limited due to the low number of couples available and the low heritability of the trait (Supplementary Table S4). In particular, as a majority of the cohort is still alive it was necessary to use parental longevity to estimate genetic effects. Although this approach has been successful in identifying genetic effects for longevity in a GWAS setting (Joshi et al. 2016), the reduction in heritability due to using a parents phenotype severely impacts the precision with which genetic values can be estimated. We would therefore suggest that these results do not provide strong evidence against assortment on heritable risk factors.

A majority of the reported estimates were consistent across both cohorts and with previous estimates, where these are available. A notable exception are the reduced 
correlations for parental longevity for father-mother pairs in the UK Biobank cohort, when compared with the same estimate in the FamiLinx cohort and correlations for same sex parent pairs in both cohorts. We suggest that this is a consequence of the limitations of the UK Biobank data. Specifically, as noted previously, the UK Biobank cohort is censored. Parents who are still alive are excluded. Such censoring will bias observed correlations downwards (Begier and Hamdan 1971). This is consistent with the lower correlations observed in the UK Biobank compared with the FamiLinx cohort, which does not suffer from such censoring. This effect is exacerbated when censoring is stronger on one of the two variables as it is the case for father-mother correlations, due to higher life expectancies for females.

We hypothesized that partner correlations in longevity could be mediated through partner correlations in disease risk. For a majority of the examined disease, partner correlations had been previously reported (Muñoz et al. 2016). Our results for disease risk are in line with those for longevity. That is, the observed partner correlations, at least partly, arise due to assortment on factors correlated across generation. Indeed, for a number of diseases, in particular hypertension, we find direct evidence for assortative mating. As the results for couples of selfreported controls were in line with those using all couples, we can exclude the possibility of direct assortment on disease status. We therefore conclude that these correlation is likely to be indirectly generated through genetic correlation between the focal trait (e.g., hypertension) and another, genetically correlated, trait or traits for which assortment happens, e.g., BMI (Robinson et al. 2017). A consequence of this model is that disease prevalence in the population may potentially be increased through indirect assortment for traits or risk factors correlated with disease (Peyrot et al. 2016). Although we find direct evidence for assortment on genetic risk factors for some disease, parental correlations for other disease lack evidence for assortment from correlations of genetic values. Parental correlations for these diseases could arise due to shared broad environment. In the particular case of lateonset disease, e.g., Alzheimer's, the observed correlations could arise as a consequence of correlations in longevity.

The cohorts used in this study have several limitations. For example, the already mentioned censoring of partners who are still alive in the UK Biobank. Another limitation is the lack of information about the year of birth of a majority of parents in the UK Biobank. However, correlations between the offspring's and parent's year of birth, where both are available, as well as replication of results on the parental disease history using the parents' year of birth, both suggest that adjusting for year of birth of the children is an acceptable, albeit not perfect, proxy for year of birth of the parents. In particular, results did not suggest that using the offspring's year of birth as a proxy introduced a substantial bias. The FamiLinx cohort, on the other hand, has a genealogical structure, potential biasing observed correlations upwards. However, the close agreement of estimates with those obtained in the UK Biobank does not suggest this is the case.

Taken together, the results suggest that the characteristics that influence mate choice lead to detectable assortment for familial disease and longevity. This assortment is only partially explained by birth cohort and the few factors chosen to reflect the social mating structure, suggesting a contribution to assortment for parental disease history and longevity of other traits, lifestyle choices or social factors shared among parents and children. Although we have not directly demonstrated that the underlying factors are transferred across generations, i.e., that the same behavioural or social factors that drive parental disease risk are also the factors underlying mate choice in the offspring, such a model presents the most canonical explanation. Although recent work has highlighted traits that are plausible candidates for direct assortative mating, e.g., height (Tenesa et al. 2015; Robinson et al. 2017), our work suggests a network of effects, whereby direct assortative mating on observable factors leads to indirect assortment for a multitude of genetically correlated traits. This highlights that assortative mating can have effects far beyond the focal trait and suggests widespread levels of pleiotropy. Understanding the contributions that mate choice and cultural transmission of behaviours and environments across generations make to these correlations will present a major but exciting challenge of future research.

\section{Data availability}

Required data can be accessed through the UK Biobank (http://www.ukbiobank.ac.uk/) and the FamiLinx website (http://www.familinx.org/), respectively. For analyses involving genotypes, we used the individuals genotyped in phase 1 of the UK Biobank genotyping project, which were released by the UK Biobank in June 2015. The genotype data were downloaded on 5 June 2015. The DISSECT software used to perform the analysis based on genetic values is freely available from http://www.dissect.ed.ac.uk/.

Acknowledgements This work was mainly supported by The Roslin Institute Strategic Grant funding from the BBSRC. AT also acknowledges funding from the Medical Research Council Human Genetics Unit. This work used the ARCHER UK National Supercomputing Service (http://www.archer.ac.uk) and the Edinburgh Compute and Data Facility (ECDF) (http://www.ecdf.ed.ac.uk/). This research has been conducted using the UK Biobank Resource under project 6684 . 


\section{Compliance with ethical standards}

Conflict of interest The authors declare that they have no conflict of interest.

Publisher's note: Springer Nature remains neutral with regard to jurisdictional claims in published maps and institutional affiliations.

\section{References}

Anonymous (1903) Assortative mating in man: a cooperative study. Biometrika 2:481-498

Begier MH, Hamdan MA (1971) Correlation in a bivariate normal distribution with truncation in both variables. Aust $\mathrm{J}$ Stat 13:77-82

Belsky DW, Domingue BW, Wedow R et al. (2018) Genetic analysis of social-class mobility in five longitudinal studies. Proc Natl Acad Sci USA 115:E7275-E7284

Bulik-Sullivan, B, Finucane HK, Anttila V et al. (2015) An atlas of genetic correlations across human diseases and traits. Nat Genet 47:1236-1241

Canela-Xandri O, Law A, Gray A et al. (2015) A new tool called DISSECT for analysing large genomic data sets using a Big Data approach Nat Commun 6:10162

Canela-Xandri O, Rawlik K, Tenesa A (2018) An atlas of genetic associations in UK Biobank. Nat Genet 50:1593-1599

Canela-Xandri O, Rawlik K, Woolliams JA et al. (2016) Improved genetic profiling of anthropometric traits using a Big Data approach PLoS ONE 11:e0166755

Conley D, Laidley T, Belsky DW et al. (2016) Assortative mating and differential fertility by phenotype and genotype across the 20th century. Proc Natl Acad Sci USA 113: 6647-6652

Cover TM, Thomas JA (2012) Elements of information theory. John Wiley \& Sons, Hoboken, New Jersey, USA

Drasgow F (1986) Polychoric and polyserial correlations. In: Kotz S and Johnson N (eds) The Encyclopedia of Statistics. John Wiley and Sons, Inc. Hoboken, New Jersey, USA

Falconer DS, Mackay TFC (1996) Introduction to quantitative genetics. Prentice Hall, Pearson

Fox J (2010) polycor: Polychoric and polyserial correlations. https:// CRAN.R-project.org/package $=$ polycor

Gianola D (1982) Assortative mating and the genetic correlation. Theor Appl Genet 62:225-231

Herskind AM, McGue M, Holm NV et al. (1996) The heritability of human longevity: a population-based study of 2872 Danish twin pairs born 1870-1900. Human Genet 97:319-323
Hippisley-Cox J, Coupland C, Pringle M et al. (2002) Married couples' risk of same disease: cross sectional study BMJ 325:636

Hugh-Jones D, Verweij KJ, Pourcain BS et al. (2016) Assortative mating on educational attainment leads to genetic spousal resemblance for polygenic scores Intelligence 59:103-108

Joshi PK, Fischer K, Schraut KE et al. (2016) Variants near CHRNA3/ 5 and APOE have age-and sex-related effects on human lifespan. Nat Commun 7:11174

Kaplanis J, Gordon A, Shor T et al. (2018) Quantitative analysis of population-scale family trees with millions of relatives. Science 360:171-175

Kong A, Thorleifsson G, Frigge ML et al. (2018) The nature of nurture: effects of parental genotypes. Science 359:424-428

Lee SH, Naomi RW, Goddard ME et al. (2011) Estimating missing heritability for disease from genome-wide association studies Am J Human Genet 88:294-305

Muñoz M, Pong-Wong R, Canela-Xandri O et al. (2016) Evaluating the contribution of genetics and familial shared environment to common disease using the UK Biobank. Nat Genet 48:980-983

Nordsletten AE, Larsson H, Crowley JJ et al. (2016) Patterns of nonrandom mating within and across 11 major psychiatric disorders. JAMA Psychiatry 73:354-361

Peyrot WJ, Robinson MR, Penninx BW et al. (2016) Exploring boundaries for the genetic consequences of assortative mating for psychiatric traits JAMA Psychiatry 73:1189-1195

Philippe P (1978) Familial correlations of longevity: an isolate-based study. Am J Med Genet 2:121-129

Robinson MR, Kleinman A, Graff M et al. (2017) Genetic evidence of assortative mating in humans. Nat Hum Behav 1:0016

Schulze R (2004) Meta-analysis-a comparison of approaches. Hogrefe \& Huber, Ashland, Ohio, USA

Silventoinen K, Kaprio J, Lahelma E et al. (2003) Assortative mating by body height and BMI: Finnish twins and their spouses Am J Human Biol 15:620-627

Stulp G, Simons MJ, Grasman S et al. (2017) Assortative mating for human height: a meta-analysis. Am J Hum Biol 29

Tenesa A, Rawlik K, Navarro P et al. (2015) Genetic determination of height-mediated mate choice Genome Biol 16:1-8

Xia C, Amador C, Huffman J et al. (2016) Pedigree- and SNPassociated genetics and recent environment are the major contributors to anthropometric and cardiometabolic trait variation. PLoS Genet 12:e1005804

Zietsch BP, Verweij KJ, Heath AC et al. (2011) Variation in human mate choice: simultaneously investigating heritability, parental influence, sexual imprinting, and assortative mating Am Nat 177:605-616 